\title{
Adipose browning response to burn trauma is impaired with aging
}

\author{
Abdikarim Abdullahi, ${ }^{1,2}$ Carly M. Knuth, ${ }^{1,2}$ Christopher Auger, ${ }^{2}$ Thibacg Sivayoganathan, ${ }^{2}$ \\ Alexandra Parousis, ${ }^{2}$ and Marc G. Jeschke $e^{1,2,3,4}$ \\ ${ }^{1}$ Faculty of Medicine, University of Toronto, Toronto, Ontario, Canada. ${ }^{2}$ Biological Sciences, Sunnybrook Research Institute, \\ Toronto, Ontario, Canada. ${ }^{3}$ Ross Tilley Burn Centre, Sunnybrook Hospital, Toronto, Ontario, Canada. ${ }^{4}$ Department of \\ Surgery, Division of Plastic Surgery, and Department of Immunology, University of Toronto, Toronto, Ontario, Canada.
}

BACKGROUND. The incidence of burn injuries in older patients is dramatically increasing as the population of older people grows. Despite the increased demand for elderly burn care, the mechanisms that mediate increased morbidity and mortality in older trauma patients are unknown. We recently showed that a burn injury invokes white adipose tissue browning that leads to a substantially increased hypermetabolic response associated with poor outcomes. Therefore, the aim of this study was to determine the effect of age on the metabolic adipose response of browning after a burn injury.

METHOD. One hundred and seventy patients with burn injury admitted to the Ross Tilley Burn Centre were prospectively enrolled and grouped by age as older ( $\geq 50$ years) and young ( $\leq 35$ years). Adipose tissue and sera were collected and analyzed for browning markers and metabolic state via histology, gene expression, and resting energy expenditure assays.

RESULTS. We found that older patients with burn injury lacked the adipose browning response, as they showed significant reductions in uncoupling protein 1 (UCP1) expression. This failure of the browning response was associated with reduced whole-body metabolism and decreased survival in older patients with burn injury. Mechanistically, we found that the adipose of both aged patients after burn trauma and aged mice after a burn showed impairments in macrophage infiltration and IL-6, key immunological regulators of the browning process after a severe trauma.

CONCLUSION. Targeting pathways that activate the browning response represents a potential therapeutic approach to improve outcomes after burn trauma for elderly patients.

FUNDING. NIH (R01-GM087285-01), Canadian Institutes of Health Research (grant no. 123336), and Canada Foundation for Innovation Leaders Opportunity Fund (no. 25407).

Conflict of interest: The authors have declared that no conflict of interest exists.

Copyright: (c) 2021, Abdullahi et al. This is an open access article published under the terms of the Creative Commons Attribution 4.0 International License.

Submitted: August 18, 2020

Accepted: July 1, 2021

Published: August 23, 2021

Reference information: /CI Insight. 2021;6(16):e143451.

https://doi.org/10.1172/jici.

insight.143451.

\section{Introduction}

According to the World Health Organization, the elderly are the fastest growing segment of the population; this population is projected to increase to 2 billion people by 2050. It is also expected that people over 65 years of age will represent almost 39\% of trauma admissions across North America by 2050 (1). This will constitute a major healthcare problem worldwide and specifically in developed countries that boast higher life expectancies. Indeed, studies have shown that older patients after burn trauma (hereafter referred to as older burn patients) have a higher risk of death and severe disability after trauma, despite having similar comorbidities and severity scores to their younger counterparts (2-4). Many believe that the increase in morbidity and mortality that is seen in older trauma patients is partially due to the substantial decline of cellular function that leads to their impaired capacity to respond to severe injuries. For instance, impaired adaptive and homeostatic mechanisms in key metabolic and immune organs in elderly people result in diminished physiological reserves and an inability to meet the heightened demands of the traumatic injury $(5,6)$. In fact, studies in older burn patients have implicated a weakened immunity and an inability to fight infections after burn injury, along with immunosenescence and altered skin morphology and thinning leading to deeper burns (7-9). Despite the recognized impairments in immunity and skin physiology in mediating poor outcomes of older trauma patients, the metabolic alterations with aging and their role in after trauma outcomes in these patients remains unclear. 
Perhaps the most serious gap in our understanding of the age-associated metabolic decline of trauma patients is the role of the adipose tissue, in particular how adipose tissue remodeling from white- to brown-like adipocytes affects energy expenditure and outcomes (10-12). Whereas white adipose tissue (WAT) is responsible for storing large amounts of triglycerides, brown adipose tissue (BAT) is characterized by smaller lipid droplets, increased mitochondrial biogenesis, and the expression of uncoupling protein 1 (UCP1), which uncouples ATP generation from oxidative phosphorylation, thus dissipating energy as heat (13-15). Unfortunately, both BAT activity and quantity have been shown to decline during aging, with diminished reserves occurring as early as the mid-40s in humans and in midlife for rodents (11). Recently, a more physiologically significant type of brown-like adipocyte that also expresses UCP1, termed beige/brite adipocytes, have been identified within WAT depots in response to $\beta$ adrenergic activation under cold conditions (16). Disease and pathological conditions, such as cancer and burn trauma, have also recently been reported to induce beige adipocyte formation and, subsequently, body weight and fat loss (17-20). Although the age-dependent decline in BAT is well characterized, whether a similar age-dependent decline in the formation of injury-induced beige adipocytes is unknown. Deciphering the age-associated changes in beige fat activation, particularly during nonphysiological states, such as burn trauma, will help us to devise new therapies that could potentially reverse the age-associated impairments in fat oxidation and fuel availability that mediate poor outcomes during the injury (21).

The aim of the following study was to examine the alterations to the browning process with aging during trauma. We report impaired beige adipocyte formation and increased mortality in both an aged murine model of thermal injury and clinical burn patients. Impairments in burn-induced browning were not a delayed response, as aged mice failed to exhibit beige adipocyte formation even up to 1 month after injury. Impaired macrophage recruitment to the WAT of both murine animals and clinical patients mediated the failure in browning in response to the burn trauma. Specifically, the two chief regulators of burn-induced browning, IL-6 and alternatively activated macrophages, were both decreased in aged mice. Thus, our findings support the notion that an age-dependent failure to form beige adipocytes is not only present in cold-induced physiological states, but also exists during conditions of injury.

\section{Results}

Failure of burn-induced beige adipocyte formation in older patients. To assess whether aging impairs the WAT browning response observed after burn trauma, we retrospectively enrolled 425 burn patients admitted to our trauma burn center (Figure 1). After assessing for eligibility, we enrolled 64 young, severely burned patients (26 \pm 6 years old) with burns encompassing $37 \% \pm 16 \%$ of their total body surface area (TBSA) (Table 1). We also recruited 110 older severely burned patients ( $63 \pm 10$ years old) with burns covering $34 \%$ $\pm 14 \%$ of their TBSA (Table 1). Young control patients without burn injury (30 \pm 4 years old) and older patients (57 \pm 8 years old) were also recruited into our study (Table 2). Subcutaneous WAT (sWAT) samples collected from enrolled patients were stratified into two groups based on age.

We found that older burn patients $(29 \%)$ had a significantly higher overall mortality rate compared with that of younger burn patients (7\%) (Figure 2A). Whole-body resting energy expenditure (REE) and lipolysis measurements also revealed that older burn patients had a reduced metabolic rate after injury compared with that of younger burn patients (Figure 2, B and C). This was further associated with a decline in adipose remodeling and beige adipocyte formation in older burn patients (Figure 2, $\mathrm{D}$ and E). Compared with sWAT from control patients without burn injury, sWAT from young burn patients stained positively for UCP1 and contained multilocular cells (Figure 2, D-F). In contrast to young patients, sWAT from older patients showed no evidence of browning following burn injury, as assessed by the lack of staining for UCP1 and the absence of multilocular cells (Figure 2, D-F). In corroboration with our immunohistochemistry data, UCP1 mRNA expression was higher in the sWAT of young burn patients relative to that in the older burn group (Figure $2 \mathrm{G}$ ). When the data were further stratified into young ( $\leq 35$ years) and elderly ( $\geq 65$ years) groups, the effects were more pronounced within the elderly subgroup, showing a greater increase in mortality and a more pronounced decrease in adipose browning (Supplemental Figure 1; supplemental material available online with this article; https:// doi.org/10.1172/jci.insight.143451DS1). Together, these findings suggest that beige adipocyte formation after a burn trauma may have therapeutic relevance and the loss of browning in older burn patients may be associated with poor outcomes. 


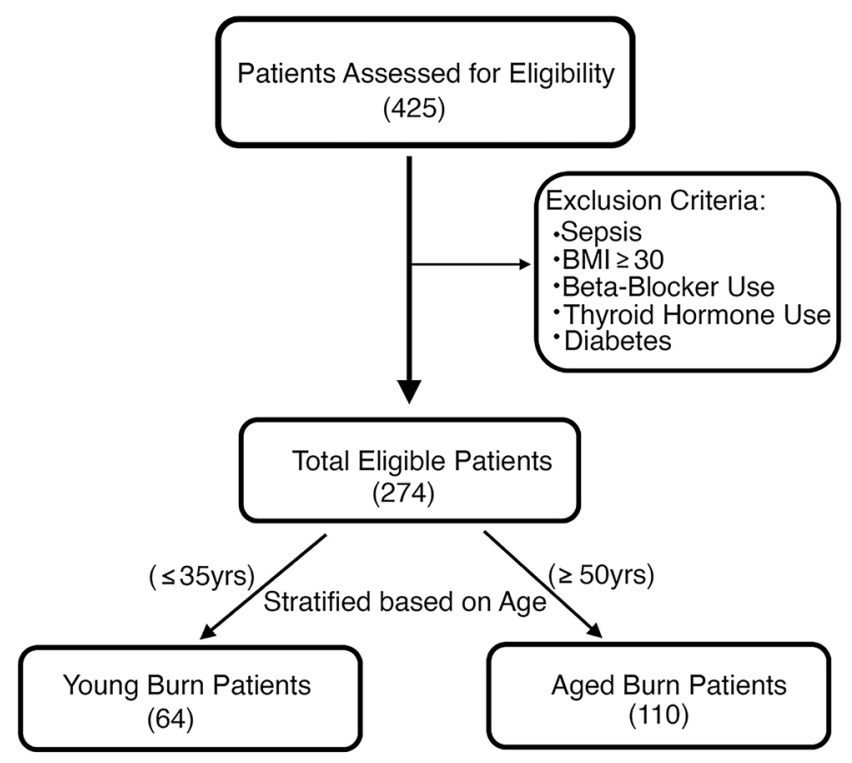

Figure 1. CONSORT flow diagram showing enrollment of patients and allocation.

Failure of burn-induced beige adipocyte formation in aged mice. To examine if the observations from our clinical elderly burn patients could be recapitulated in a murine model, we next examined whether trauma-induced browning was also impaired in aged mice. To do this, we utilized a well-characterized animal burn injury model whereby C57BL/6J mice that were either 8 weeks old (young mice) or 52 weeks old (aged mice) and were then randomized into either the control or 30\% TBSA burn injury group (Supplemental Figure 2) (22). Similar to the increased mortality we observed in elderly burn patients, aged mice subjected to a severe burn injury also showed an increase in mortality relative to their younger counterparts (Figure 3A). This increased mortality in aged mice after burn injury was also associated with a reduced metabolic response, as assessed by the lack of tissue wasting and fat metabolism in these mice (Figure 3, B and C). Consistent with adipose remodeling after burn injury and increased fat metabolism, analysis of sWAT from young mice showed reduced lipid droplet size and increased multilocular adipocytes compared with those in aged mice (Figure 3, D-G). Furthermore, young mice exposed to a burn trauma formed beige adipocytes and expressed the key browning marker UCP1 within the inguinal sWAT depot (Figure 4, A-C). Conversely, aged mice exposed to a burn trauma failed to form beige adipocytes and exhibited a blunted induction of the browning gene, UCP1 (Figure 4, A-C). Additionally, the chief regulator of mitochondrial biogenesis peroxisome proliferator-activated receptor $\gamma$ coactivator $1 \alpha(\mathrm{PGC1A})$ and mitochondrial respiration were also increased in young mice and blunted in aged mice after burn injury, further confirming an impairment in browning in aged mice (Figure 4, D and E). We further assessed whether the impaired browning response to burn trauma in aged mice persisted throughout the course of the injury past the 1 week time point. Indeed, while young mice 2 weeks after burn trauma still showed signs of browning, such as UCP1 $1^{+}$beige adipocytes and increased mitochondrial respiration, these responses failed to develop in the aged mice at this time point (Supplemental Figure 3, A-C). This blunted adipose browning response observed in aged mice persisted even after 1 month after burn trauma compared with that in their younger counterparts (Supplemental Figure 4, A-C). Thus, these findings further advance the notion that aging impairs the adipose browning response to trauma and that aged mice fail to recover this potentially therapeutic metabolic process, even as the injury manifests over time.

Key regulators of burn-induced browning are diminished in both aged mice and older burn patients. Recently, the cytokines IL-6, IL-4 and alternatively activated macrophages that secrete norepinephrine have been implicated in mediating beige adipocyte formation during burn trauma, cancer, calorie restriction, and cold-induced WAT browning $(18,23-27)$. As such, we hypothesized that aging also interferes with the expression of the aforementioned key regulators required to form beige adipocytes in response to burn trauma. To test this hypothesis in humans, we first assessed the recruitment of macrophages to 
Table 1. Demographics of patients after burn injury

\begin{tabular}{|c|c|c|c|}
\hline & Young burn patients $(n=64)$ & Older burn patients $(n=110)$ & $P$ value \\
\hline Age $(y r)$, mean \pm SEM & $26 \pm 6$ & $63 \pm 10$ & $P>0.0001$ \\
\hline \multicolumn{4}{|l|}{ Sex } \\
\hline Female, no. (\%) & $15(23 \%)$ & $30(27 \%)$ & NS \\
\hline \multicolumn{4}{|l|}{ Race } \\
\hline Asian, no. (\%) & $9(14 \%)$ & $15(13 \%)$ & - \\
\hline Other/unknown & $10(16 \%)$ & $13(12 \%)$ & - \\
\hline \multicolumn{4}{|l|}{ Burn etiology } \\
\hline Flame, no. (\%) & $52(81 \%)$ & $68(62 \%)$ & - \\
\hline Scald, no. (\%) & $12(19 \%)$ & $42(38 \%)$ & - \\
\hline TBSA (\%), mean \pm SEM & $37 \pm 16$ & $34 \pm 14$ & NS \\
\hline Cholesterol (mmol/L), mean \pm SEM & $2.6 \pm 0.7$ & $2.8 \pm 0.8$ & NS \\
\hline LDL (mmol/L), mean \pm SEM & $1.4 \pm 0.9$ & $1.2 \pm 0.5$ & NS \\
\hline
\end{tabular}

Age, race, and sex data presented as mean and total percentage relative to total population enrolled. All other data points represent mean \pm SEM and were analyzed by unpaired $t$ test, as described in Methods.

the adipose tissue, a critical first step in the adipose browning process, as we have previously shown (23). We found both a decrease in granulocyte colony-stimulating factor (GCSF), the key chemokine involved in macrophage chemotaxis, and the number of infiltrated $\mathrm{CD} 11 \mathrm{~b}^{+}$macrophages in the sWAT of older burn patients compared with that in their younger counterparts (Figure 5, A and B). Additionally, older burn patients showed decreased plasma levels of the key cytokines (IL-4, IL-6, IL-13) and norepinephrine, which have been shown to regulate WAT browning in burns $(23,24)$ (Figure $5, \mathrm{C}-\mathrm{F}$ ). Similarly, in response to burn injury, aged mice showed an impaired macrophage migratory capability, which was associated with a decrease in the number of infiltrated $\mathrm{F} 4 / 80^{+}$macrophages within sWAT (Figure 6, A-D). Not only did the aged mice show impairments in macrophage migration and recruitment, but they also showed decreases in circulating IL-6 levels, alternatively activated macrophages, and norepinephrine, which are all requirements for the activation of the browning process during a burn trauma $(23,24)$ (Figure $6, \mathrm{E}-\mathrm{G})$. All together, these findings suggest that the disruption of the adipose browning response to trauma with aging is, at least in part, mediated by reduced macrophage recruitment, deficiencies in key cytokines, and the subsequent norepinephrine secretion required to initiate the browning process.

\section{Discussion}

Metabolic adaptation occurs after a severe trauma in order to better optimize nutrient usage. This metabolic response that includes adipose browning is critical to the hypermetabolic response that is activated after a burn trauma in order to drive host survival. Several lines of evidence have demonstrated that the dysregulation of whole-body energy expenditure and substrate oxidation can have dire effects on the metabolic health and disease outcomes of elderly patients (28-30). Specifically, changes in adipose tissue physiology and function that control whole-body energy homeostasis have been implicated in mediating altered metabolism with aging $(5,8,11)$. Older patients after burn trauma are at a significantly increased risk for complications and death as a result of degenerative changes in metabolism (e.g., dwindling energy expenditure, mitochondrial function, accumulating adiposity, and substantial decline in muscle mass) $(3,8,31)$. The present work has uncovered some important features of WAT remodeling in response to trauma during aging. Here, we found an age-related impairment in the formation of beige adipocytes after injury in patients and aged mice, which was associated with increased mortality following a burn trauma. 
Table 2. Demographics of control patients without burn injury

\begin{tabular}{|c|c|c|c|}
\hline & Young patients without burn $(n=7)$ & Older patients without burn $(n=8)$ & $P$ value \\
\hline Age $(y r)$, mean \pm SEM & $30 \pm 4$ & $57 \pm 6$ & $<0.0001$ \\
\hline \multicolumn{4}{|l|}{ Sex } \\
\hline Female, no. (\%) & $3(43 \%)$ & $3(37 \%)$ & NS \\
\hline \multicolumn{4}{|l|}{ Race } \\
\hline Asian, no. (\%) & $2(29 \%)$ & $3(38 \%)$ & - \\
\hline Other/unknown, no. (\%) & $2(29 \%)$ & $1(12 \%)$ & - \\
\hline \multicolumn{4}{|l|}{ Preadmission comorbidities } \\
\hline BMI, mean \pm SEM & $25.2 \pm 3.1$ & $27 \pm 2.8$ & NS \\
\hline Diabetes, no. & 0.0 & 0.0 & - \\
\hline Liver failure, no. & 0.0 & 0.0 & - \\
\hline
\end{tabular}

Age, race, and sex data presented as mean and total percentage relative to total population enrolled. All other data points represent mean \pm SEM and were analyzed by unpaired $t$ test, as described in Methods.

We further found a failure to activate adipose browning in response to burn trauma in aged mice at as early as 1 week; this impairment remained even after 1 month after injury. Interestingly, we found a decrease in key contributors to beige adipocyte formation in response to trauma, including diminished macrophage adipose infiltration and a parallel decrease in IL-6 in aged mice and older patients. Thus, our data suggest that therapeutically restoring adipose browning could potentially improve both the metabolic state and overall outcome in aged burn trauma patients.

Previous studies have predominantly focused on the aging effects on BAT, leaving the age-related changes in beige adipocyte formation largely unexplored. Indeed, only two other studies have identified age-related changes in browning, with both studies yielding similar results to ours presented here (32, 33). For instance, it has been recently reported that cold-induced beige adipocyte formation is impaired both in aged mice and patients (32). Similarly, another recent study found that female mice have a progressive age-dependent loss of sWAT browning, and this process runs in parallel with morphological changes in BAT (33). Our studies differ in that we are the first to our knowledge to assess the effects of aging on WAT browning in the context of an injury and not in a physiological context, such as cold exposure. These variations between our studies and those previously reported are important as the physiological and cellular activities activated during cold exposure and in trauma or cancer, where browning has been reported, are starkly different $(16,18,23)$. Furthermore, while we have previously reported that chronic browning in adult burn patients fuels persistent hepatic steatosis and hypermetabolism, this should not suggest that all beige activity is bad per se $(34,35)$. We believe that a certain amount of beige activity may be beneficial in burns by helping to mobilize energy reserves to establish adequate wound healing and protective immune responses. Indeed, our findings herein, in which reduced beige adipocyte activity with aging was associated with poorer outcomes after a burn injury, are not contradictory to our previous findings. Rather, our studies suggest that activation of WAT browning in either extreme (too little vs. too much) are problematic for patient outcomes. Ultimately, further research is required to enable more nuanced monitoring of this browning process in burns during aging so that we can better identify the optimal activation levels of this remarkable process for its therapeutic potential.

Complementary studies that have utilized cold exposure models to induce browning have leant credence to the notion that age-dependent beige progenitor dysfunction might, in part, be attributed to cellular senescence (32). For example, genetic and pharmacologic manipulation of p38/MAPK and the Ink4a/Arf pathway, 2 key senescence regulators, was sufficient to block cold-inducible beige adipocyte formation in young mice (36). Conversely, genetic and pharmacological inhibition of the Ink4a/Arf pathway blunted senescence in older animals and restored metabolic benefits of cold-induced browning $(32,37)$. However, the age-related changes to the key regulators of WAT browning outside of senescence have not been studied. This is particularly important, as most of the literature on browning has implicated both macrophages and the cytokines IL- 4 and IL- 6 in the formation of beige adipocytes (23-27). Our potentially novel findings 


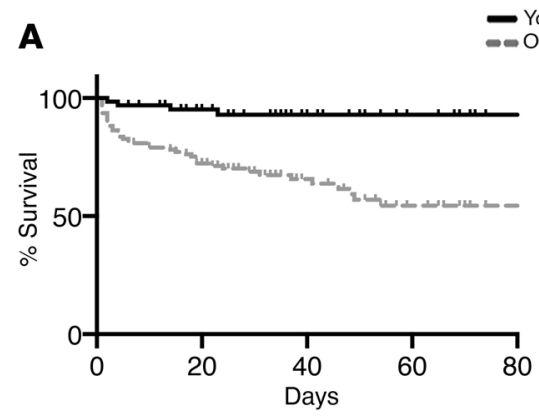

Young Burn Patients Old Burn Patients

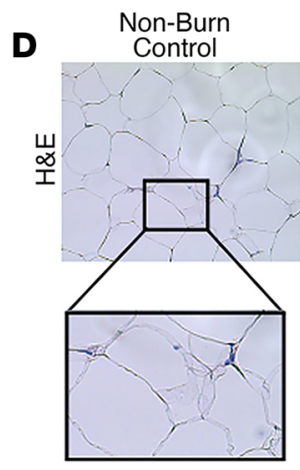

Young Burn
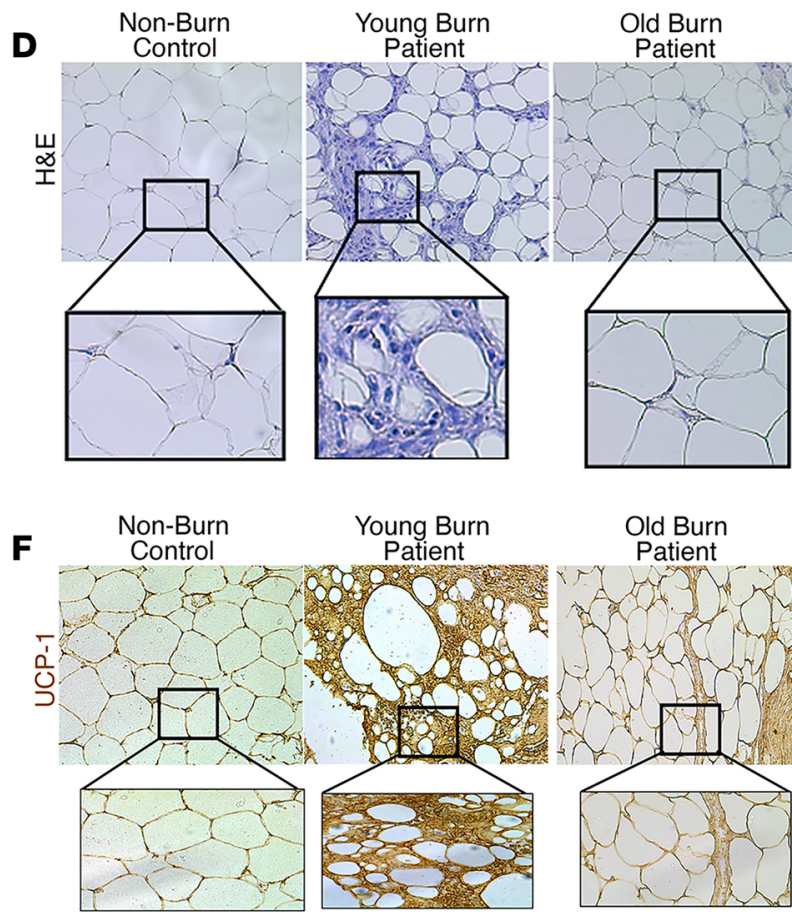

Young Burn Patient

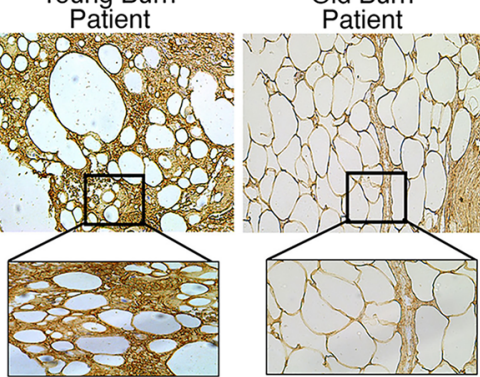

B

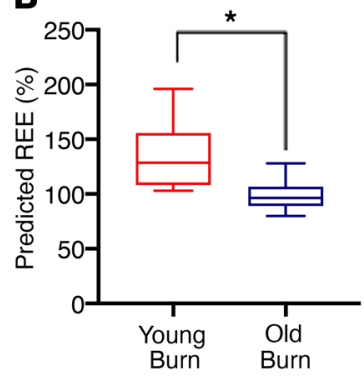

C

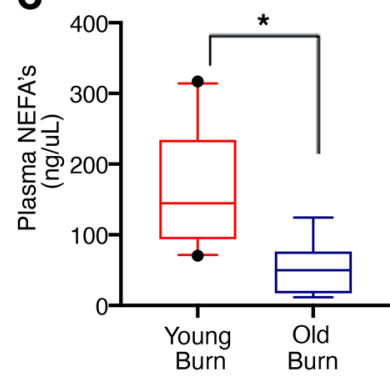

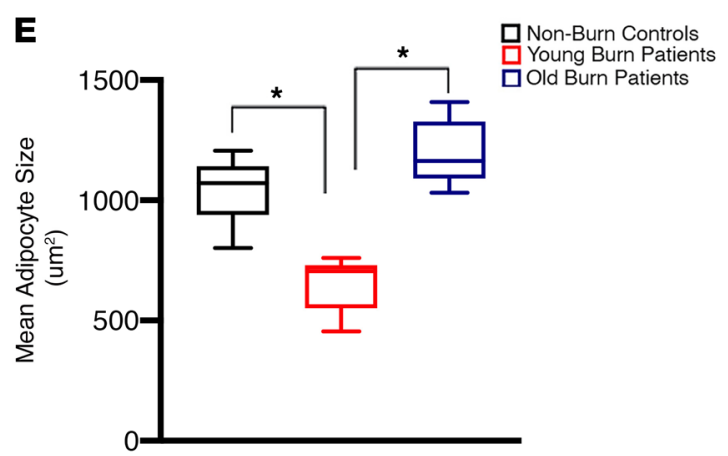

G

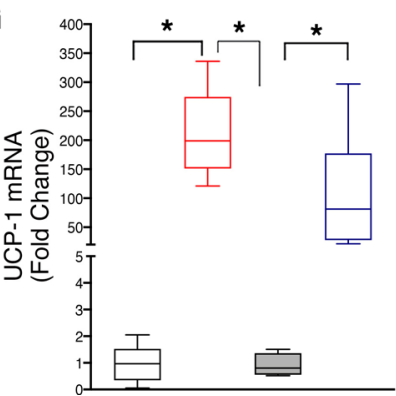

Non-Burn Controls Young Burn Patients Non-Burn Old Controls

Figure 2. Failure of burn-induced beige fat formation in aged burn patients. (A) Kaplan-Meier survival curves of young and older burn patients with burns over $20 \%$ of total body surface area. (B) Box-and-whisker plots showing average measured and predicted REE in young and older burn patients. (C) Box-and-whisker plots showing plasma concentration of free fatty acids in young and older burn patients. (D) Immunohistochemistry H\&E staining of subcutaneous WAT of young and older burn patients. (E) Quantification of average lipid droplet size in subcutaneous WAT isolated from young and older burn patients. (F) Immunohistochemistry staining of UCP1 in subcutaneous WAT of young and older burn patients. (G) Box-and-whisker plots showing quantitative RT-PCR analysis of browning gene UCP1 in subcutaneous WAT isolated from young and older burn patients. The box plots depict the 5\%-95\% quartiles (whiskers), the upper and lower quartiles, and the median. The length of the box represents the interquartile range. Data represent the mean \pm SEM. ${ }^{*} P<0.05$, young vs. older burn patients $(n=55-107)$. Scale bars: $100 \mu$ m. Statistical differences were determined using an unpaired $t$ test with Welch's correction.

in rodents and clinical burn patients have demonstrated impairments in macrophage migration and IL-6 production, necessary factors for trauma-induced browning. This suggests that the age-dependent failure in browning is not only due to senescence of adipocytes, but also changes in the regulators of browning as well. Thus, pharmacologic or other approaches that could modulate macrophage activity and IL-4/IL-6 production in the adipose tissue during aging could be exploited therapeutically in the recovery of beige fat to potentially improve metabolic health, as been done in the field of obesity $(38,39)$.

In summary, our findings indicate that aging results in a profoundly reduced adipose browning response to burn trauma and that this is associated with significantly worse outcomes. Subsequent questions to be investigated include whether these findings are also true in other hypermetabolic conditions, such as cancer, where adipose browning has also been reported. Further understanding of the regulation and alterations of the WAT browning metabolic response with aging in conditions outside of cold exposure will provide new directions and possibly targeted therapies to improve the metabolic health status of older trauma patients. 

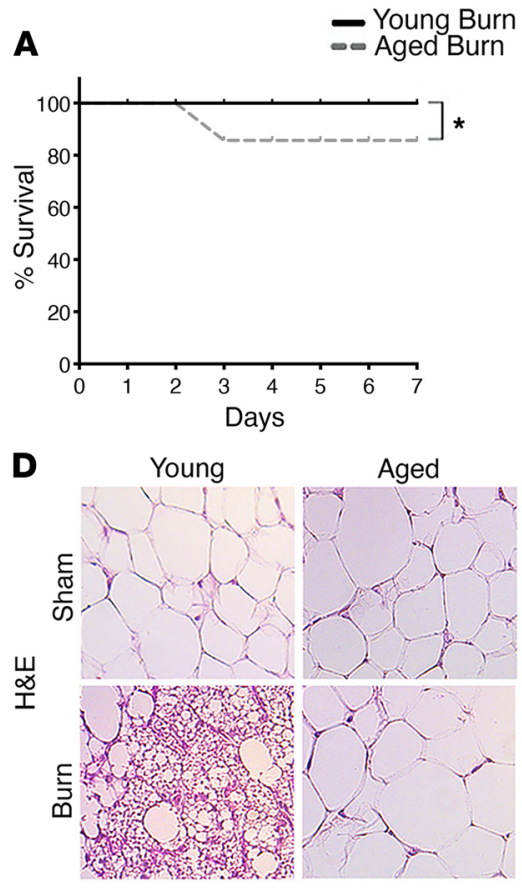

$\mathbf{F}$

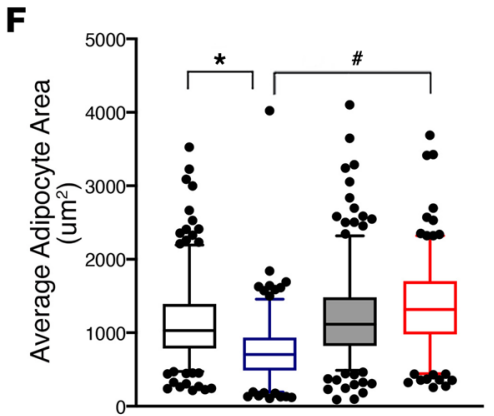

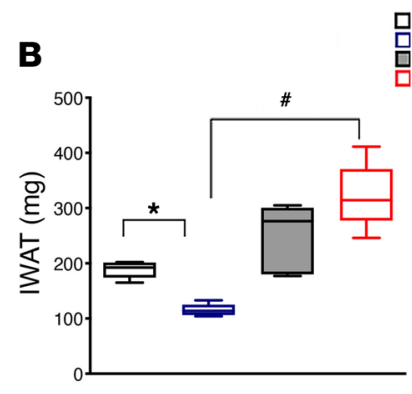
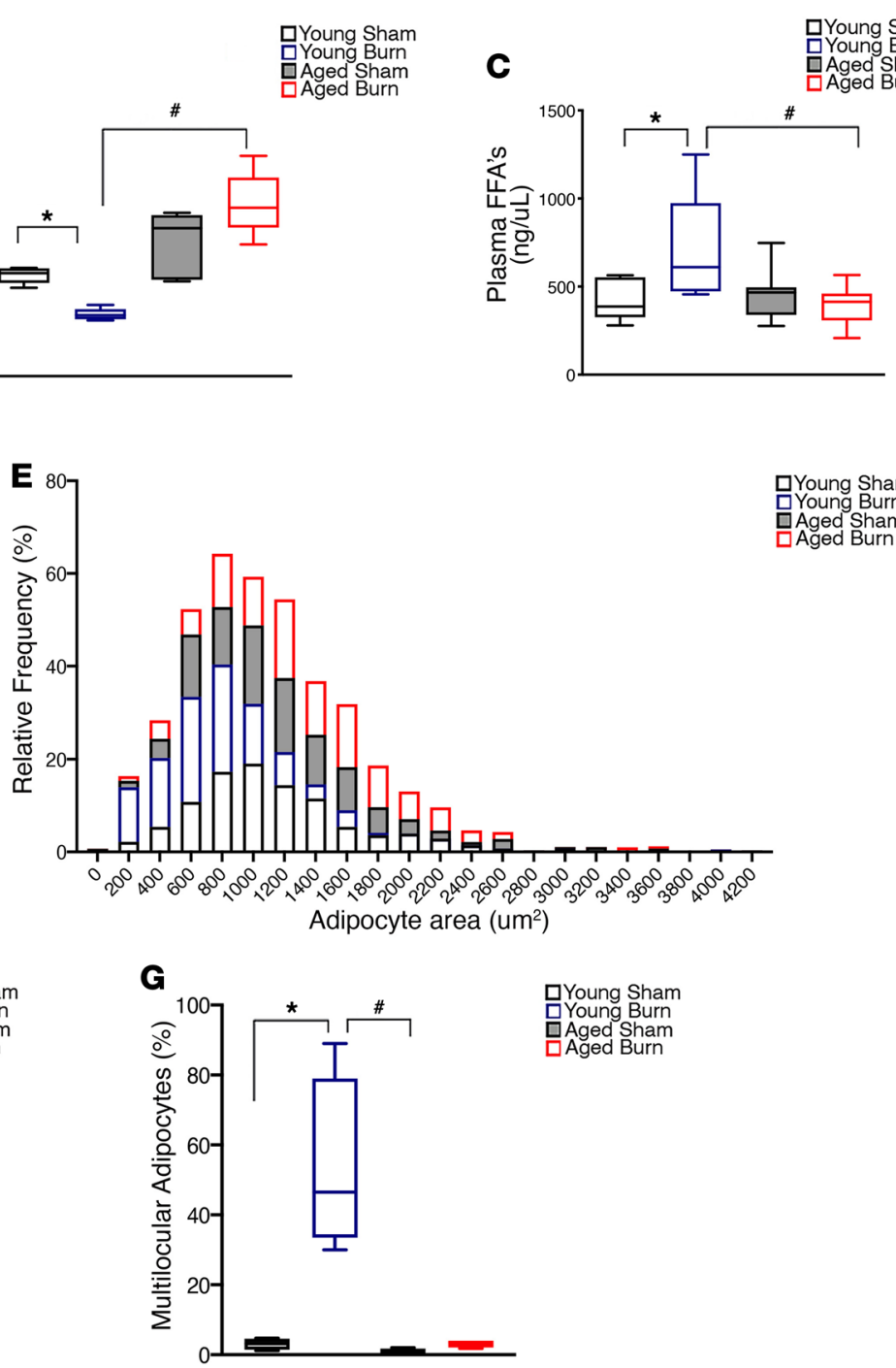

Figure 3. Increased mortality and impaired adipose remodeling in aged mice after a burn injury. (A) Kaplan-Meier survival curves of young vs. aged mice subjected to a 30\% total body surface area burn injury. (B) Changes in inguinal WAT mass in young and aged mice 1 week after burn injury. (C) Plasma concentration of free fatty acids in young and aged mice 1 week after burn injury. (D) Immunohistochemistry H\&E staining of subcutaneous WAT of young and aged mice. (E) Different lipid droplet size frequency in subcutaneous WAT isolated from young and aged mice. (F) Quantification of average lipid droplet size in subcutaneous WAT isolated from young and aged mice. (C) Quantification of the number of multilocular adipocytes in subcutaneous WAT isolated from young and aged mice. Data represent the mean \pm SEM. ${ }^{*} P<0.05$, vs. sham; ${ }^{*} P<0.05$, vs. burn $(n=10)$. Scale bars: $100 \mu$ m. Statistical differences were determined using 2-way ANOVA followed by Bonferroni post hoc test.

\section{Methods}

Human samples. We retrospectively enrolled 425 burn patients admitted to our trauma burn center. After assessing for eligibility based on our exclusion criteria (sepsis, BMI $\geq 30$, beta blocker use, thyroid hormone use, and diabetes), those patients that remained were stratified into two age groups; young burn patients ( $26 \pm 6 \mathrm{yr})$ and older burn patients (63 $\pm 10 \mathrm{yr})$ (Supplemental Figure 1). The age cutoff chosen for the older burn patient cohort ( $>50 \mathrm{yr}$ ) was selected based on previous studies that reported a significant decline in WAT browning and brown fat after this age $(29,32,33)$. sWAT was collected from the torso or lower/upper limbs of all patients enrolled, depending on the type of burn injury sustained. Adipose tissue was only collected from patients who had surgeries that occurred $\geq 10$ days after their injury, as previous studies have shown browning is only evoked after this time period $(19,20)$. Tissue samples collected were immediately transferred to the laboratory and either frozen in liquid nitrogen $\left(-80^{\circ} \mathrm{C}\right)$ or placed in fixative for histology until analysis. REE was measured with a Sensor Medics 2900 metabolic measurement cart. 

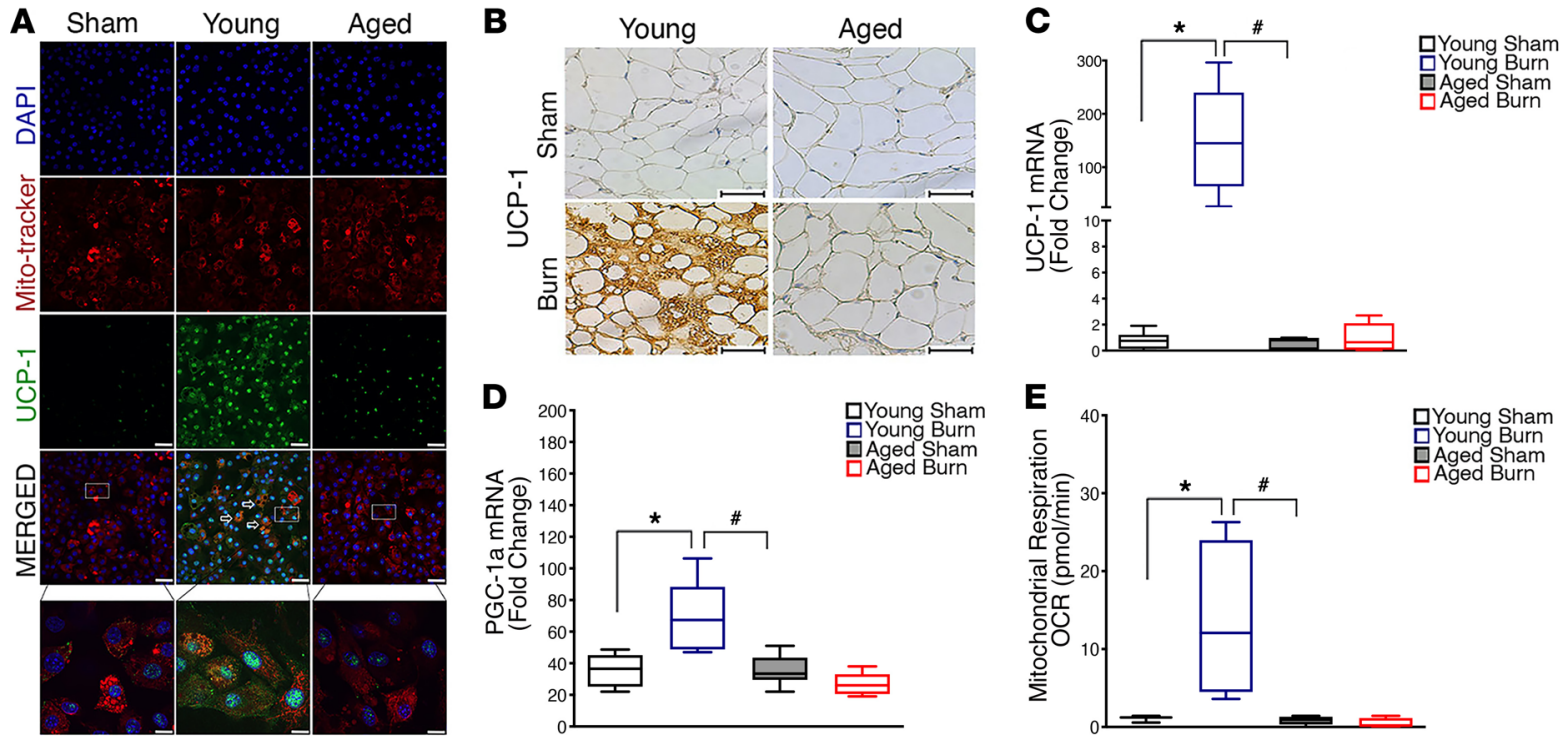

Figure 4. Failure of burn-induced beige fat formation in aged mice. (A) Differentiated isolated adipocytes were fixed and then stained for MitoTracker (Red), UCP1 (Green), and nuclei (DAPI) and then imaged (original magnification, $\times 60$ ). (B) Immunohistochemistry staining of UCP1 in inguinal WAT of young and aged mice 1 week after burn injury. (C) Quantitative RT-PCR analysis of browning gene UCP1 in inguinal WAT of young and aged mice 1 week after burn injury. (D) Quantitative RT-PCR analysis of mitochondrial biogenesis gene PGC1 $\alpha$ in inguinal WAT of young and aged mice 1 week after burn injury. (E) Analysis of mitochondrial oxygen consumption rate in isolated inguinal WAT of young and aged mice 1 week after burn injury. Data represent the mean \pm SEM. ${ }^{*} P<0.05$, vs. sham; ${ }^{*} P<0.05$, vs. burn $(n=6-9)$. Scale bars: $100 \mu$ m. Statistical differences were determined using 2-way ANOVA followed by Bonferroni post hoc test.

Animals. Male C57BL/6J mice that were 6 weeks or 1 year old were purchased from The Jackson Laboratory (stock no. 000664 ) and housed at ambient temperatures of approximately $23^{\circ} \mathrm{C}-24^{\circ} \mathrm{C}$. Mice were cared for in accordance with the Guide for the Care and Use of Laboratory Animals (National Academies Press, 2011). After arrival, mice were acclimated to our facility for 2 weeks before the commencement of the experiments. By the date of experiments, the mice were either 8 weeks old (young mice) or 52 weeks old (aged mice).

Mouse thermal injury model. We used a previously well-characterized rodent burn injury model (22). Mice received a 30\% TBSA full-thickness burn. All mice were anesthetized with $2.5 \%$ isoflurane and shaved along the dorsal spine region. Ringer's lactate $(2-3 \mathrm{~mL})$ was injected subcutaneously in all treatment mice to protect the spine, and buprenorphine $(0.05-0.1 \mathrm{mg} / \mathrm{kg}$ body weight) was injected before and after the burn injury when necessary, as required for pain management. A full-thickness third-degree dorsal scald burn encompassing $15 \%$ TBSA and a $15 \%$ wound on the ventral surface was induced by immersing the dorsum of the mice in $98^{\circ} \mathrm{C}$ water for 10 seconds and the ventral region for 2 seconds. Mice were subsequently housed individually in sterile cages and fed ad libitum until sacrifice. Sham (control) mice underwent identical experimental procedures, with the exception of the burn injury. WAT (inguinal, epididymal) and plasma were collected upon sacrifice and stored in $-80^{\circ} \mathrm{C}$ until analysis.

Histology and immunohistochemistry. Adipose tissues were immediately fixed in 10\% formalin and then maintained in $70 \%$ ethanol before paraffin embedding. Subsequently, tissues were sectioned and stained with $\mathrm{H} \& \mathrm{E}$ or incubated with UCP1 (U6382, MilliporeSigma), F4/80 (MF48000AbD, Thermo Fisher), or CD11c (ab52632, Abcam) antibody followed by DAB staining. Imaging was performed on a LSM confocal microscope (Zeiss).

Quantitative PCR. Total RNA isolated from adipose tissues was analyzed by quantitative RT-PCR. Briefly, RNA was isolated from tissues using TRIzol-chloroform (Life Technologies) with subsequent purification using the RNeasy Kit (Qiagen) according to the manufacturer's instructions. RNA (2 mg) was transcribed to cDNA using the high-capacity cDNA reverse transcription kit (Applied Biosystems). Real-time quantitative PCR was performed using the Applied Biosystems Step One Plus Real-Time PCR System. Primer sequences are available in Supplemental Table 1.

Primary macrophage culture and conditioned medium preparation. Mice were sacrificed by cervical dislocation and were rinsed in $70 \%$ (vol/vol) ethanol. Then, bone marrow was isolated from femurs and tibias. Bone marrow cells were plated at a density of $1 \times 10^{6}$ to $2 \times 10^{6}$ cells $/ \mathrm{mL}$ in RPMI-1640 medium (supplemented with $10 \% \mathrm{FCS}, 1 \%$ glutamine, $1 \%$ penicillin-streptomycin, and $10 \mathrm{ng} / \mathrm{mL}$ macrophage 
A

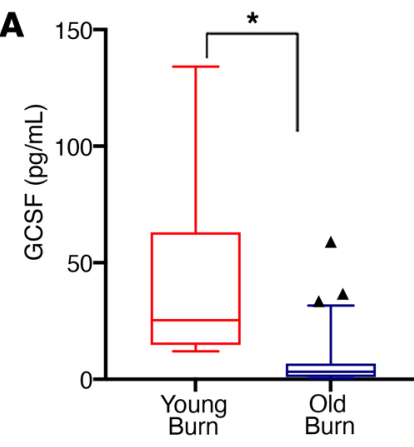

C

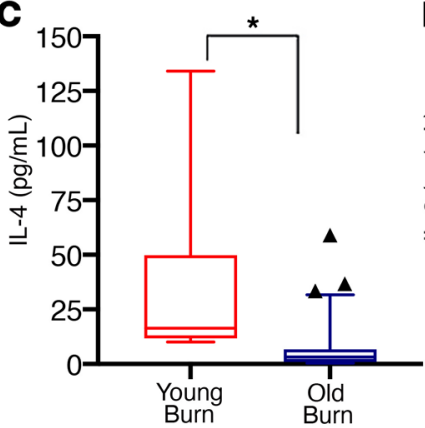

B

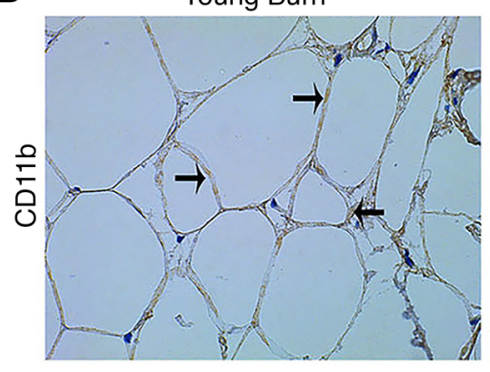

D

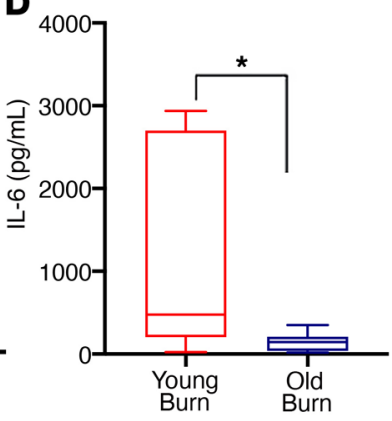

E

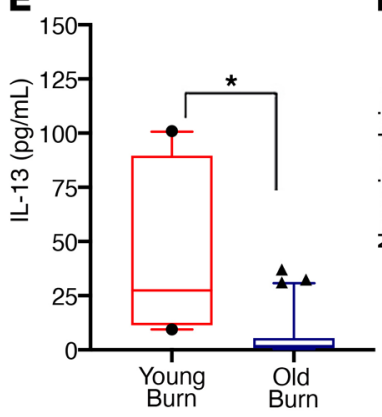

Old Burn

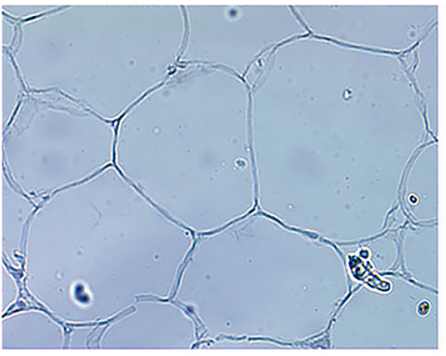

$\mathbf{F}$

Figure 5. Blunted macrophage recruitment and decreased expression of cytokines involved in the regulation of burn-induced browning in older patients. (A) Box-and-whisker plots showing expression levels of the key protein GCSF involved in the mobilization of macrophages in the plasma of young and older burn patients. (B) Immunohistochemistry staining of macrophage marker CD11b in subcutaneous WAT isolated from young and older burn patients. Arrows indicate CD11b+ cells. (C) Box-and-whisker plots showing plasma concentration of IL-4 in young and older burn patients. (D) Box-and-whisker plots showing plasma concentration of IL-6 in young and older burn patients. (E) Box-and-whisker plots showing plasma concentration of IL-13 in young and older burn patients. (F) Norepinephrine content in sWAT isolated from young and older burn patients. The box plots depict the 5\%-95\% quartiles (whiskers), the upper and lower quartiles, and the median. The length of the box represents the interquartile range. Data represent the mean \pm SEM. ${ }^{*} P<0.05$, young vs. older burn patients $(n=15-30)$. Scale bars: $50 \mu \mathrm{m}$. Statistical differences were determined using an unpaired $t$ test with Welch's correction.

colony-stimulating factor [PeproTech]) on 6-well plates and were allowed to differentiate for 7 days. After differentiation, these BMDMs were used for an in vitro scratch assay.

Cytokine profile. Rodent and human sera were collected, and a panel of cytokines was measured using a Luminex platform (MilliporeSigma).

Primary adipocyte culture. Isolated mouse SV cells were cultured in Dulbecco's modified Eagle medium with $10 \%$ FBS (Invitrogen) at $37^{\circ} \mathrm{C}$ in a $5 \% \mathrm{CO}_{2}$ environment. Beige adipogenesis was induced by treating confluent cells with DMEM containing 10\% FBS, $0.5 \mathrm{mM}$ isobutylmethylxanthine, $125 \mathrm{nM}$ indomethacin, $1 \mathrm{mM}$ dexamethasone, $850 \mathrm{nM}$ insulin, $2 \mathrm{nM} \mathrm{T3}$, and $1 \mathrm{mM}$ rosiglitazone (Alexis Biochemicals). Differentiated adipocytes were then stained with MitoTracker Red (Thermo Fisher), UCP1 (U6382, MilliporeSig$\mathrm{ma})$, and DAPI (BD Biosciences).

Respiration assays. Oxygen consumption rates were measured in freshly excised adipose tissues (inguinal adipose depots). Tissues were minced in mitochondrial isolation buffer (MHSE + BSA; $210 \mathrm{mM}$ mannitol, $70 \mathrm{mM}$ sucrose, $5 \mathrm{mM}$ HEPES, $1 \mathrm{mM}$ EGTA, 0.5\% [w/v] fatty acid-free BSA, $\mathrm{pH} 7.2)$. The tissue was then homogenized using a Teflon glass homogenizer. Mitochondria were isolated via differential centrifugation. Briefly, the homogenate was centrifuged at $600 \mathrm{~g}$ for 10 minutes, and the supernatant decanted into a new tube. This fraction was centrifuged at $9000 \mathrm{~g}$ for 10 minutes to obtain a mitochondrial pellet, which was subsequently resuspended in $50 \mu \mathrm{L}$ MHSE + BSA. BCA assays (Thermo Scientific) were performed to gauge protein concentrations. Mitochondrial bioenergetics were assessed using a Seahorse XF96 analyzer (Agilent Technologies). Mitochondrial respiration in a coupled state $(10 \mu \mathrm{g} / \mathrm{well})$ was measured in mitochondrial assay solution (220 mM mannitol, $70 \mathrm{mM}$ sucrose, $10 \mathrm{mM} \mathrm{KH}_{2} \mathrm{PO}_{4}, 5 \mathrm{mM} \mathrm{MgCl}$, $2 \mathrm{mM} \mathrm{HEPES}, 1 \mathrm{mM}$ EGTA, and $0.2 \%[\mathrm{w} / \mathrm{v}]$ fatty acid-free BSA, $\mathrm{pH} 7.2$, at $\left.37^{\circ} \mathrm{C}\right)$ containing succinate as a substrate $(10 \mathrm{mM})$ and rotenone $(2 \mu \mathrm{M})$. State 3 respiration (phosphorylating respiration) was triggered via the injection of a cocktail containing $4 \mathrm{mM}$ ADP along with $10 \mathrm{mM}$ pyruvate, $2.5 \mathrm{mM}$ glutamate, and $2.5 \mathrm{mM}$ malate. State $4 \mathrm{o}$ respiration was assessed by the addition of $2.5 \mu \mathrm{g} / \mathrm{mL}$ oligomycin, while maximal uncoupler-stimulated 
A

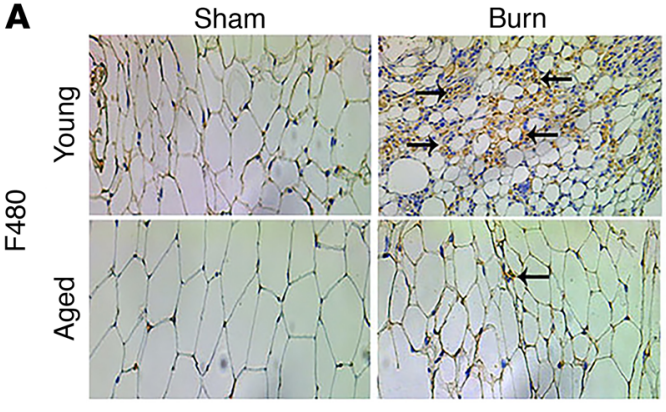

Day 0

C

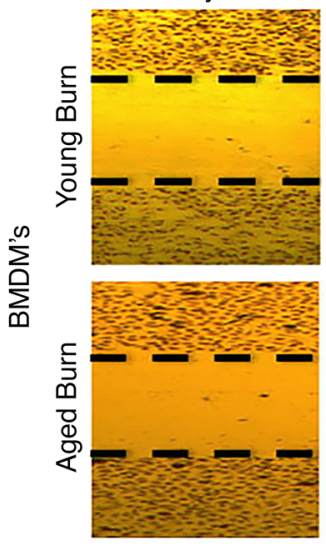

Day 3

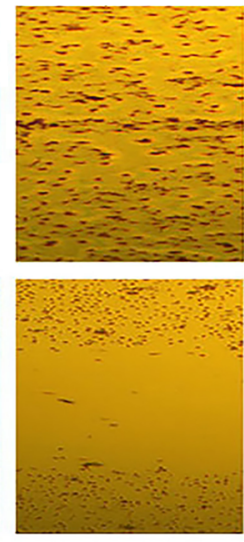

$\mathbf{B}$

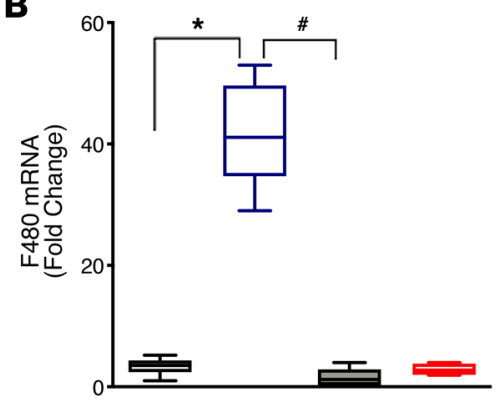

GYoung Sham CYoung Burn aged Burn
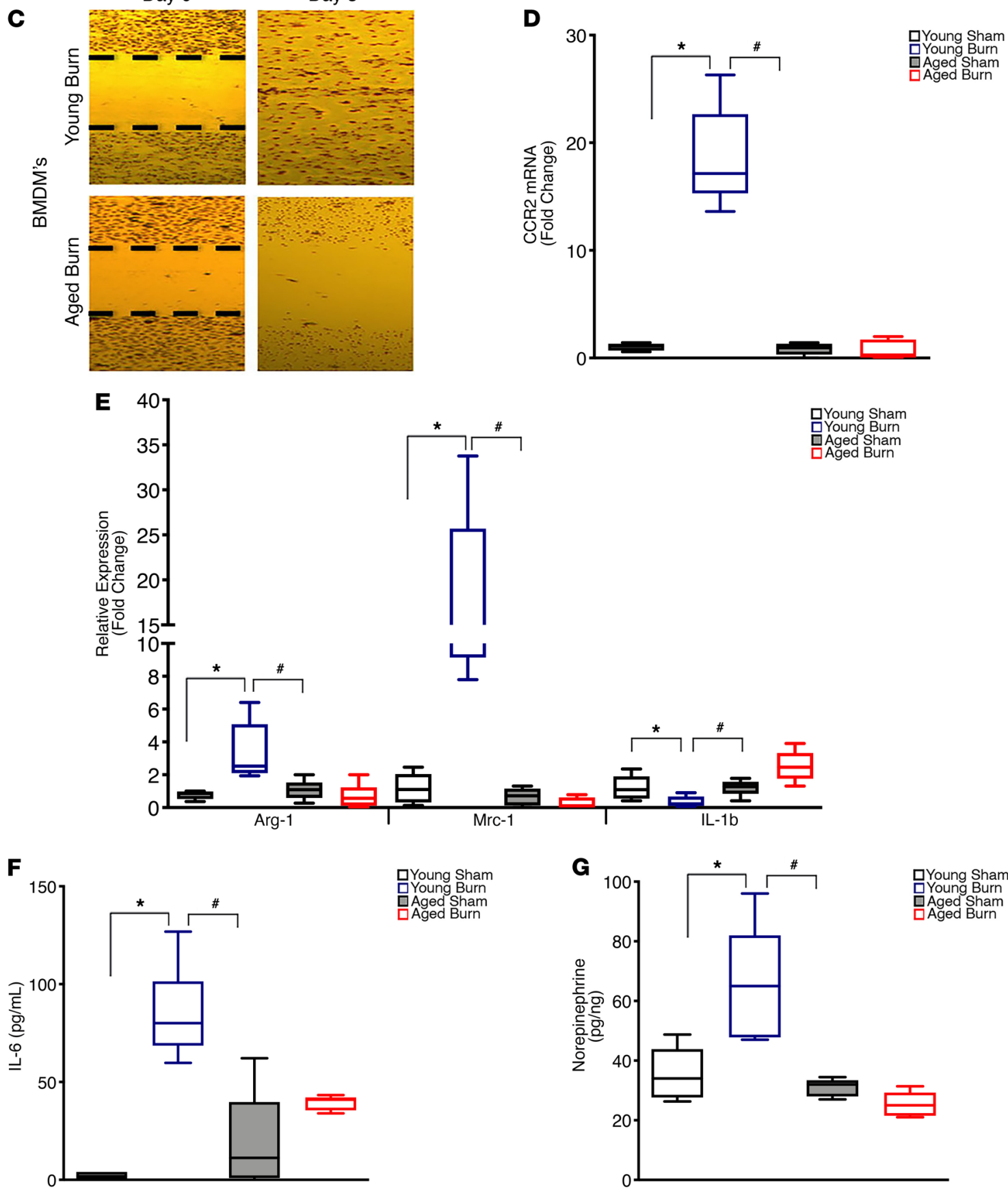

Figure 6. Aging impairs macrophage chemotaxis and the secretion of cytokines involved in the regulation of burn-induced browning in mice. (A) Immunohistochemistry staining of macrophage marker F4/80 in inguinal WAT of young and aged mice 1 week after burn injury. (B) Quantitative RT-PCR analysis of macrophage marker F4/80 in inguinal WAT of young and aged mice 1 week after burn injury. (C) In vitro chemotaxis assay of bone marrow-derived 
macrophages isolated from young and aged mice 1 week after burn injury. (D) Quantitative RT-PCR analysis of macrophage chemotaxis marker CCR2 in inguinal WAT of young and aged mice 1 week after burn injury. (E) Quantitative RT-PCR analysis of M2 macrophage markers in inguinal WAT of young and aged mice 1 week after burn injury. (F) Plasma concentration of IL-6 in young and aged mice 1 week after burn injury. (G) Norepinephrine content in inguinal WAT of young and aged mice 1 week after burn injury. Data represent the mean \pm SEM. ${ }^{*} P<0.05$, vs. sham; ${ }^{\#} P<0.05$, vs. burn $(n=10)$. Scale bars: $100 \mu$ m. Statistical differences were determined using 2-way ANOVA followed by Bonferroni post hoc test.

respiration was observed following the injection of $4 \mu \mathrm{M}$ carbonyl cyanide 4-(trifluoromethoxy)phenylhydrazone (FCCP). Antimycin A (4 $\mu \mathrm{M})$, a complex III inhibitor, was added at the end of the experiment to inhibit mitochondrial respiration. The Seahorse XF Wave software was used to analyze the data.

Lipolysis assay. For in vivo lipolysis assays in mice, serum samples were collected, and free fatty acid levels were then quantified per the manufacturer's instructions (Biovision Abcam). Serum fatty acids from patients were analyzed by gas chromatography-mass spectrometry performed by the Analytical Facility for Bioactive Molecules platform at the Hospital for Sick Children (Toronto, Ontario, Canada). Briefly, serum samples $(20 \mu \mathrm{L})$ were spiked with an internal standard mix and acidified with $\mathrm{HCl}$. Nonesterified fatty acids were acidified and double extracted with hexane. The fatty acids were then converted to their pentafluorobenzyl esters using $1 \%$ pentafluorobenzyl bromide/diisopropylamine (1:1) and separated by automated gas chromatography (GC Agilent 7890A, Agilent Technologies) on a fused-silica SP2380 capillary column $(30 \mathrm{~m} \times 0.25 \mathrm{~mm} \times 0.2 \mu \mathrm{m}$ film thickness; Supelco Analytical). Fatty acid ions were detected and measured using a MSD Agilent 5975C quadrupole mass detector (Agilent Technologies). Peaks of fatty acid esters were identified by comparisons with individual fatty acid standards (Supelco Analytical).

Catecholamine assay. Norepinephrine was quantified using an ELISA kit (BAE-5200R, Rocky Mountain Diagnostics) per the manufacturer's protocols. For norepinephrine ELISAs, tissues were homogenized by sonication in homogenization buffer $\left(0.01 \mathrm{~N} \mathrm{HCl}, 1 \mathrm{mM}\right.$ EDTA, $\left.4 \mathrm{mM} \mathrm{Na} \mathrm{S}_{2} \mathrm{O}_{5}\right)$, and cellular debris was pelleted by centrifugation at $15,115 \mathrm{~g}$ for 15 minutes at $4^{\circ} \mathrm{C}$. The cleared homogenates were collected and stored in a $-80^{\circ} \mathrm{C}$ freezer before quantification. All samples were normalized to total tissue protein concentration.

Statistics. All data are presented as mean \pm SEM and were analyzed using Prism 9 (GraphPad). Normality and equal variance were verified in all data. Data were $\log$ transformed if normal distribution and/or equal variance tests failed. Statistical differences between 2 groups were evaluated using 2-tailed unpaired $t$ test with Welch's correction, where appropriate, for single variables. Statistical differences among 3 or more groups were evaluated using 2-way ANOVA followed by Bonferroni post hoc test. Results were considered significant at $P<0.05$.

Study approval. Patients admitted to the Ross Tilley Burn Centre at Sunnybrook Health Sciences Centre (Toronto, Ontario, Canada) and patients without burn undergoing elective surgery preoperatively provided consent for tissue and blood collection. Approval for our human study was obtained from the Research Ethics Board at Sunnybrook Health Sciences Centre. All animal studies and procedures were approved by the Sunnybrook Research Institute Animal Care Committee under AUP 467.

\section{Author contributions}

AA performed the experiments, analyzed the data, and wrote the manuscript. CMK and CA performed the experiments, analyzed the data, and edited the manuscript. TS and AP performed the experiments. MGJ edited the manuscript and conceptualized the paper.

\section{Acknowledgments}

AA is a recipient of and supported by the Banting Postdoctoral Fellowships program through the Canadian Institutes of Health Research. This work was supported by grants from the Canadian Institutes of Health Research (grant no. 123336), the Canada Foundation for Innovation Leaders Opportunity Fund (no. 25407), and the NIH (R01GM087285, 5R01GM133961-02).

Address correspondence to: Marc G. Jeschke, Ross Tilley Burn Centre, Sunnybrook Health Sciences Centre, Division of Plastic Surgery, Department of Surgery, University of Toronto, 2075 Bayview Ave., Rm D704, Toronto, Ontario M4N 3M5, Canada. Phone: 416.480.6703; Email: marc.jeschke@sunnybrook.ca. 
1. Llompart-Pou JA, et al. Severe trauma in the geriatric population. World J Crit Care Med. 2017;6(2):99-106.

2. Keller JM, et al. Geriatric trauma: demographics, injuries, and mortality. J Orthop Trauma. 2012;26(9):e161-e165.

3. Tornetta P, et al. Morbidity and mortality in elderly trauma patients. J Trauma. 1999;46(4):702-706.

4. Sharfman ZT,et al. Increased morbidity and mortality in elderly patients with lower extremity trauma and associated injuries: a review of 420,066 patients from the national trauma database. Injury. 2021;52(4):757-766.

5. Frankenfield D, et al. Age-related differences in the metabolic response to injury. J Trauma. 2000;48(1):49-56.

6. Rehou S, et al. Acute phase response in critically ill elderly burn patients. Crit Care Med. 2019;47(2):201-209.

7. Goei H, et al. Burns in the elderly: a nationwide study on management and clinical outcomes. Burns Trauma. 2020;8:tkaa027.

8. Jeschke MG, et al. Pathophysiologic response to burns in the elderly. EBioMedicine. 2015;2(10):1536-1548.

9. Jeschke MG, et al. State of the science burn research: burns in the elderly. J Burn Care Res. 2020;41(1):65-83.

10. Saito M. Brown adipose tissue as a regulator of energy expenditure and body fat in humans. Diabetes Metab J. 2013;37(1):22-29.

11. Rossato M. Aging and brown adipose tissue activity decline in human: does the brain extinguish the fire? Aging Clin Exp Res. 2016;28(3):579-581

12. Matsushita M, et al. Impact of brown adipose tissue on body fatness and glucose metabolism in healthy humans. Int J Obes (Lond). 2014;38(6):812-817.

13. Abdullahi A, Jeschke MG. White adipose tissue browning: a double-edged sword. Trends Endocrinol Metab. 2016;27(8):542-552.

14. Sidossis L, Kajimura S. Brown and beige fat in humans: thermogenic adipocytes that control energy and glucose homeostasis. J Clin Invest. 2015;125(2):478-486.

15. Chondronikola M, Sidossis LS. Brown and beige fat: from molecules to physiology. Biochim Biophys Acta Mol Cell Biol Lipids. 2019;1864(1):91-103.

16. Barbatelli G, et al. The emergence of cold-induced brown adipocytes in mouse white fat depots is determined predominantly by white to brown adipocyte transdifferentiation. Am J Physiol Endocrinol Metab. 2010;298(6):E1244-E1253.

17. Kir S, et al. Tumour-derived PTH-related protein triggers adipose tissue browning and cancer cachexia. Nature. 2014;513(7516):100-104.

18. Petruzzelli M, et al. A switch from white to brown fat increases energy expenditure in cancer-associated cachexia. Cell Metab. 2014;20(3):433-447.

19. Patsouris D, et al. Burn induces browning of the subcutaneous white adipose tissue in mice and humans. Cell Rep. 2015;13(8):1538-1544.

20. Sidossis LS, et al. Browning of subcutaneous white adipose tissue in humans after severe adrenergic stress. Cell Metab. 2015;22(2):219-227.

21. Johnson AA, Stolzing A. The role of lipid metabolism in aging, lifespan regulation, and age-related disease. Aging Cell. 2019;18(6):e13048.

22. Abdullahi A, et al. Animal models in burn research. Cell Mol Life Sci. 2014;71(17):3241-3255.

23. Abdullahi A, et al. Alternatively activated macrophages drive browning of white adipose tissue in burns. Ann Surg. 2019;269(3):554-563.

24. Abdullahi A, et al. IL-6 signal from the bone marrow is required for the browning of white adipose tissue post burn injury. Shock. 2017;47(1):33-39.

25. Lee MW, et al. Activated type 2 innate lymphoid cells regulate beige fat biogenesis. Cell. 2015;160(1-2):74-87.

26. Qiu Y, et al. Eosinophils and type 2 cytokine signaling in macrophages orchestrate development of functional beige fat. Cell. 2014;157(6):1292-1308.

27. Fabbiano S, et al. Caloric restriction leads to browning of white adipose tissue through type 2 immune signaling. Cell Metab. 2016;24(3):434-446.

28. Barzilai N, et al. The critical role of metabolic pathways in aging. Diabetes. 2012;61(6):1315-1322.

29. Manini TM. Energy expenditure and aging. Ageing Res Rev. 2010;9(1):1-11.

30. Henry CJ. Mechanisms of changes in basal metabolism during ageing. Eur J Clin Nutr. 2000;54(suppl 3):S77-S91.

31. Auger C, et al. Hepatic mitochondrial bioenergetics in aged C57BL/6 mice exhibit delayed recovery from severe burn injury. Biochim Biophys Acta Mol Basis Dis. 2017;1863(11):2705-2714.

32. Berry DC, et al. Cellular aging contributes to failure of cold-induced beige adipocyte formation in old mice and humans. Cell Metab. 2017;25(2):481.

33. Goncalves LF, et al. Ageing is associated with brown adipose tissue remodelling and loss of white fat browning in female C57BL/6 mice. Int J Exp Pathol. 2017;98(2):100-108.

34. Abdullahi A, et al. Browning of white adipose tissue after a burn injury promotes hepatic steatosis and dysfunction. Cell Death Dis. 2019;10(12):870.

35. Abdullahi A, Jeschke MG. Taming the flames: targeting white adipose tissue browning in hypermetabolic conditions. Endocr Rev. 2017;38(6):538-549.

36. Bordicchia M, et al. Cardiac natriuretic peptides act via p38 MAPK to induce the brown fat thermogenic program in mouse and human adipocytes. J Clin Invest. 2012;122(3):1022-1036.

37. Rabhi N, et al. Cdkn2a deficiency promotes adipose tissue browning. Mol Metab. 2018;8:65-76.

38. Hanssen MJ, et al. Short-term cold acclimation recruits brown adipose tissue in obese humans. Diabetes. 2016;65(5):1179-1189.

39. Saito M. Brown adipose tissue as a therapeutic target for human obesity. Obes Res Clin Pract. 2013;7(6):e432-e438. 